DOI: 10.20472/IAC.2018.043.003

DR. HANAN ALMAZROUEI

United Arab Emirates, United Arab Emirates

\title{
EXPATRIATE MANAGERIAL ADJUSTMENT IN THE UNITED ARAB EMIRATES: ANTECEDENTS AND CONSEQUENCES
}

\begin{abstract}
:
International workforce mobility is worldwide. Expatriates bring skills and new perspectives that can enhance organizational performance. Dealing positively with these workers is important to improve their performance, particularly given the risk of maladjustment they may experience from a new culture.
\end{abstract}

The purpose of this study is to (a) determine the influences contributing to expatriate adjustment in the UAE, (b) ascertain constructive influences such as social networking, work and life satisfaction, and personal growth, and undesirable influences such as stress and adjustment issues, and (c) identify how employers and communities can better support expatriates.

We will produce two versions of the findings. The first will be an extended version containing interview summaries, survey results and conclusions based on our hypotheses. The second will take the form of an executive summary useful to practitioners, and containing recommendations for expatriates, HR professionals and related personnel. This summary will assist organizations to better understand the experiences of expatriates, enabling the introduction of better supporting frameworks.

In summary, we will use our findings and recommendations to develop awareness of the issues expatriates face and encourage more effective use of the resources and perspectives they bring. For example, the results of this study may be useful as a counterpoint to the policy of Emiratization which seeks to limit the hiring of foreign workers, even those with valuable skills, knowledge or experience. Expatriates in the UAE or other Middle Eastern nations and prospective expatriates, managers and HR practitioners in organizations employing expatriates, UAE government policy developers and community leaders will all find the outcomes of this study beneficial.

The study is quantitative in nature, employing between 350 and 550 questionnaires. Intended participants, to be selected by random sampling, would be targeted based on their non-Arab background, language difficulties, and their length of tenure in their UAE organization. A pilot survey of approximately 50 to 75 questionnaires will be used to improve the research instrument's validity and reliability. Government representatives will be included as they are the intended beneficiaries of this research, thus enhancing the possibility of including in government policy the insights gained. Various media platforms such as websites can be used to disseminate the results to potential expatriates and their employers as a component of the outreach program. University facilities will be use to conduct the study. This research will be strengthened through gaining feedback at conferences within the next two years, with a view to subsequently publishing the findings.

\section{Keywords:}


Expatriate, adjustment, UAE

JEL Classification: M16 